\section{Demonstration of creatine phosphokinase in human myocardial slices}

R. J. TRICKEY AND M. J. DAVIES Department of Histopathology, St George's Hospital Medical School, London SWI7 ORE, UK

In myocardial infarction progressive enzyme loss occurs from the necrotic area of muscle. The demonstration of enzyme activity in the normal muscle may enable the area of infarction to be demonstrated as an area of diminished or absent activity. Nachlas and Schnitka (1963) demonstrated dehydrogenase activity by use of a tetrazolium salt (nitro-blue tetrazolium; NBT) which is reduced to a coloured formazan by $\mathrm{H}+$ ions. The substrate for dehydrogenase may be either contained within the myocardium or added to the incubation medium as lactate, malate, or succinate. The latter technique is usually necessary to produce a colour reaction in postmortem material when more than 24 hours have elapsed since death. The dehydrogenase reactions can be used to demonstrate and accurately delineate human myocardial infarction of more than 12 hours' duration. Creatine phosphokinase (CPK) leaks more rapidly from damaged myocardial cells

Received for publication 3 October 1978 and is the enzyme often estimated in serum to indicate myocardial necrosis in clinical practice.

Demonstration of CPK activity in the myocardium provides a means of identifying recent myocardial infarction in a manner analogous to the NBT method for dehydrogenases.

The methods used to assay CPK activity for the auto-analyser (Siegel and Cohen, 1967; Savigano et al., 1969) can be modified to produce a colour reaction from NBT in tissue slices.

\section{Methods}

One-centimetre slices of fresh myocardium are washed briefly in physiological saline to remove excess blood and incubated at $37^{\circ} \mathrm{C}$ for 15 minutes in each medium.

INCUBATION MEDIUM 1

Imidazole buffer, 0.1 M, pH $6.8 \quad 100 \mathrm{ml}$

Adenosine 5-diphosphate sodium salt $250 \mathrm{mg}$

Creatine phosphate $\quad 300 \mathrm{mg}$

Adenosine 5-monophosphate $251 \mathrm{mg}$

INCUBATION MEDIUM 2

Phosphate buffer, pH 7.0 $100 \mathrm{ml}$

NBT $(1 \mathrm{mg} / \mathrm{ml}) \quad 50 \mathrm{ml}$

NADP

Glucose

Fig. 1 Slice of dog myocardium 8 hours after ligation of left circumflex coronary artery. An irregular pale area of muscle has not developed the deep colour shown in the remainder of the myocardium. 
The slices are agitated or turned at regular intervals. During the second incubation the slice is watched continuously, and the reaction is stopped by immersion in $10 \%$ formol saline when colour has developed.

The method was tested on fresh slices of human myocardium and on an experimental model of myocardial infarction in the dog after ligation of the left circumflex coronary artery. Dogs were killed at intervals of 1-48 hours after coronary artery ligation. Slices were examined within minutes of the animal's death, after keeping the heart for 24 hours and 48 hours at $4^{\circ} \mathrm{C}$, and after 12 and 24 hours at $16^{\circ} \mathrm{C}$.

\section{Results}

Both human and dog myocardial slices develop a deep purple colour in the second incubation medium. Areas of infarction develop this colour (Fig. 1) at a reducing rate for up to 48 hours of age, after which little colour develops even on longer incubation in the second medium. Stopping the colour development by immersion in $10 \%$ formol saline allows recognition of areas of infarction. Slices kept at $4^{\circ} \mathrm{C}$ for up to 48 hours show identical reactions; slices kept at $16^{\circ} \mathrm{C}$ for 12 hours develop irregular colour, and after 24 hours at $16^{\circ} \mathrm{C}$ no colour may develop.

\section{Discussion}

The method demonstrates both experimental and human myocardial infarction. Experimental infarction is demonstrated at 6 to 8 hours and somewhat earlier than conventional NBT methods for dehydrogenases.

The reactions involved in the development of a colour reaction are shown in Figure 2. All limiting reagents are added in the incubation media except CPK, hexokinase, and glucose-6-phosphate dehydrogenase. In tissue slices it is not practicable to add the large molecular enzymes. The reaction may therefore test a combination of all three enzymes. The final step in incubation medium 2 when used alone will develop a blue colour after longer periods, presumably due to inherent dehydrogenase activity. Adenosine monophosphate is added to the initial incubation mixture since it enhances the subsequent speed of colour development.

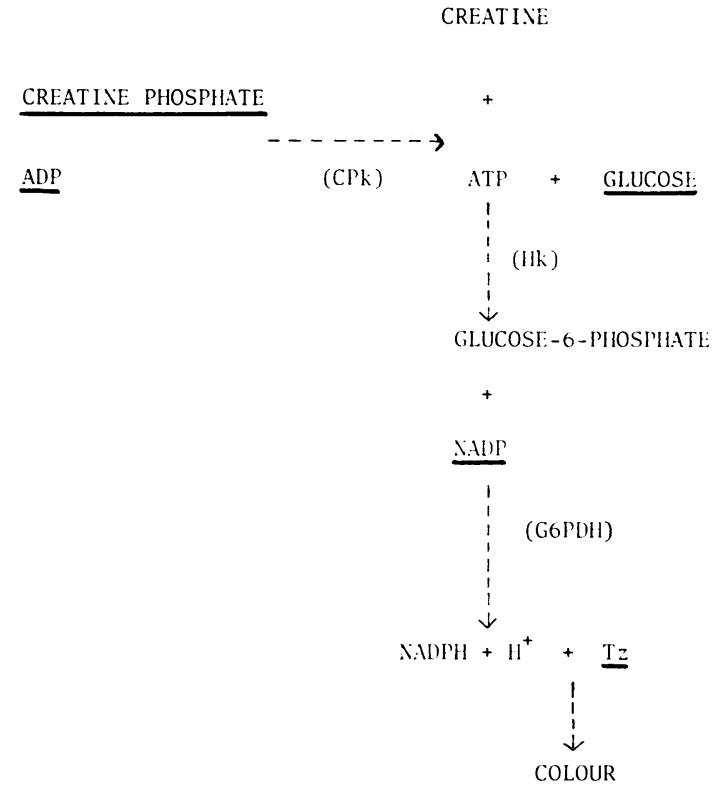

Fig. 2 Reactions involved in colour development. Substances underlined are added to the incubation media. Enzymes in brackets.

It cannot be claimed that the method is proven as a specific demonstration of CPK activity but it is an empirical method for use in the demonstration of recent muscle necrosis.

\section{References}

Nachlas, M. M., and Schnitka, T. K. (1963). Macroscopic identification of early myocardial infarcts by alterations in dehydrogenase activity. American Journal of Pathology, 42, 379-405.

Savigano, T., Hanok, A., and Kuo, J. (1969). Creatine phosphokinase activity-a study of normal and abnormal levels. American Journal of Clinical Pathology, 51, 76-85.

Siegel, A. L., and Cohen, P. S. (1967). An automated determination of creatine phosphokinase. In Automation in Analytical Chemistry (Technicon Symposium, 1966). Vol. 1, p. 474. Mediad, White Plains, N.Y.

Requests for reprints to: Professor M. J. Davies, Department of Histopathology, St George's Hospital Medical School, Cranmer Terrace, Tooting, London SW17 ORF UK. 